\title{
EI TLCAN, Un Balance a 20 Años de Vigencia y desde lo Procesal
}

\author{
Eusebio F. Flores Barraza ${ }^{1}$ \\ Yesenia Gastelum Ortega ${ }^{2}$
}

\section{Sumario: I Introducción. II Resoluciones de los paneles arbitrales del TLCAN- NAFTA a 10 años. III Conclusiones de las decisiones arbitrales.}

Resumen.- En el presente trabajo encontraremos un breve recorrido histórico, producto de las decisiones de los tribunales arbitrales que han resuelto en 10 años de del NAFTA (aunque ya cumplió 21 años) ; lo que nos muestra un buen indicador de: hacia dónde vamos y cómo vamos en el país con respecto a USA y Canadá

Abstract.- Inside this dissertation we find a brief historical product of the decisions of arbitral tribunals that have been resolved in 10 years of NAFTA (although, it turned 21); which shows us a good indicator about: where we're going and how we're going in our country respect to USA and Canada

Résumé.- Au present travail nous trouverons un petit voyage histotrique, produit des desisions des tribunaux arbitraux qu'ils ont résolus dans 10 ans du NAFTA (maintenant, il a 21ans); ce qui nous montre un bon indicateur de: où nous allons et comment nous allons en notre pay par rapport à les États-Unis et Canada.

Palabras clave.- Decisiones de los paneles arbitrales, Tratado: TLCAN- NAFTA.

Key words.- Decisions of the arbitration panel, Treaty: NAFTA.

Mots-clés.- Desisions des panneaux arbitraux, Traité: TLCAN-NAFTA

\footnotetext{
${ }^{1}$ Docente-Investigador de TC de la Universidad de Sonora, Doctor en Derecho por la Universidad de La Habana Profesor Investigador de Tiempo Completo del Departamento de Derecho de la Universidad de Sonora, doctoreusebioippson@live.com.mx

${ }^{2}$ Docente-Investigador de TC de la Universidad de Sonora,Candidata a Doctora por el Centro de Estudios Superiores en Ciencias Jurídicas y Criminológicas Unidad de Enlace Sonora.
} 


\section{Introducción}

El $1^{\circ}$ de Enero de 1994, fue la entronización de dos sucesos que sacudieron el país, por una parte la denominada la declaración formal de la guerrilla Zapatista en el sureste mexicano, por hechos que después serian acogidos a una ley de amnistía posterior. Igualmente como segundo suceso en esa misma fecha, la entrada en vigor del Tratado de Libre Comercio de América del Norte (TLCANNAFTA), mismo acuerdo que fue negociado entre los tres países de América: Canadá, USA y nuestro país. Desde nuestra perspectiva veremos de manera sucinta como ha impactado durante 20 años este acuerdo comercial de bienes y servicios y específicamente cuantos fallos de los paneles arbitrales se han dado y como se resolvieron para los tres países litigantes haciendo especial énfasis en nuestro país.

Un sector de economistas piensa que ha sido un éxito, mientras que otro cree que en nuestro país ha impactado el acuerdo comercial de manera negativa, sin embargo desde el punto de vista jurídico y particularmente de los fallos jurídicos poco se ha explorado. De ahí que consideramos trascendental para esta investigación hacer un breve análisis jurídico del sentido de las resoluciones a efecto de contrastar directamente esto con sus contrapartes mexicanos. Es un terreno árido en el cual nos envolveremos habida cuenta de la poca difusión que se le da y la información confidencial que dentro de los procesos se maneja. Es de particular relevancia el portal del secretariado oficial1, que publica en línea las consideraciones respectivas y que nos muestra la información relevante.

Estos mismos: "datos duros y fríos" de información habrá que sistematizarla y compilarla para efecto de darle cuerpo a nuestro trabajo. Por ultimo como en estos casos se dice cuento con ayuda de mucha información pero los yerros son 
solamente privilegio del suscrito.

Antes de analizar las resoluciones de los paneles arbitrales, es conveniente saber a groso modo cuales son los principales mecanismos de solución de controversias que se encuentran pactados en el TLCAN:

I. Capítulo XI: Inversión ${ }^{3}$, sobre mecanismos de solución en materia de servicios Financieros

II. Capítulo XIX $\mathrm{X}^{4}$ : Revisión y solución de controversias en materia de cuotas antidumping y compensatorias, Sobre casos que involucren "Prácticas desleales de comercio Internacional" (cuyo plazo para resolver esta en 315 días)

III. Capítulo $\mathrm{XX}^{5}$ : Disposiciones institucionales $\mathrm{y}$ procedimientos para la solución de controversias, en materia de diferencias que se susciten en la Interpretación o Aplicación del acuerdo (plazo de 5 meses para laudo)

Interpretación o Aplicación del acuerdo (plazo de 5 meses para laudo)

Cuando se inicia el tratado hace 20 años, se le había apostado a una trasparencia en los procesos y controversias de los paneles arbitrales, donde se apreciaba que estábamos en igualdad de circunstancias y por supuesto un -piso plano- para los tres miembros, sin embargo desde la óptica de los mecanismos de solución de controversias (MASC) y su utilización real en estos últimos 10 años, observamos

\footnotetext{
${ }^{3}$ Secretarido del Tratado del Libre Comercio para América Ltina TLCLAN[online] Disponible en :https://www.nafta-sec-alena.org/Inicio/Textos-juridicos/Tratado-de-Libre-Comercio-de-América-delNorte?mvid=1\&secid=539c50ef-51c1-489b-808b-9e20c9872d25

${ }^{4}$ Ibid

${ }^{5}$ Ibidem
} 
una postura más complicada y menos ilusa, el autor a través de varias diapositivas analiza los fallos que se han suscitado en este periodo y concluye lo que inexorablemente arrojan los datos de 53 fallos que aparecen poco desglosados en el portal de NAFTA, los que a pesar de estar en línea no son verdaderamente muy claros. A continuación se hace un resumen en las diferentes láminas que componen el presente ensayo:

II. Resoluciones de los Paneles Arbitrales del TLCAN- NAFTA, a 10 años desde la agenda procesal de los MASC

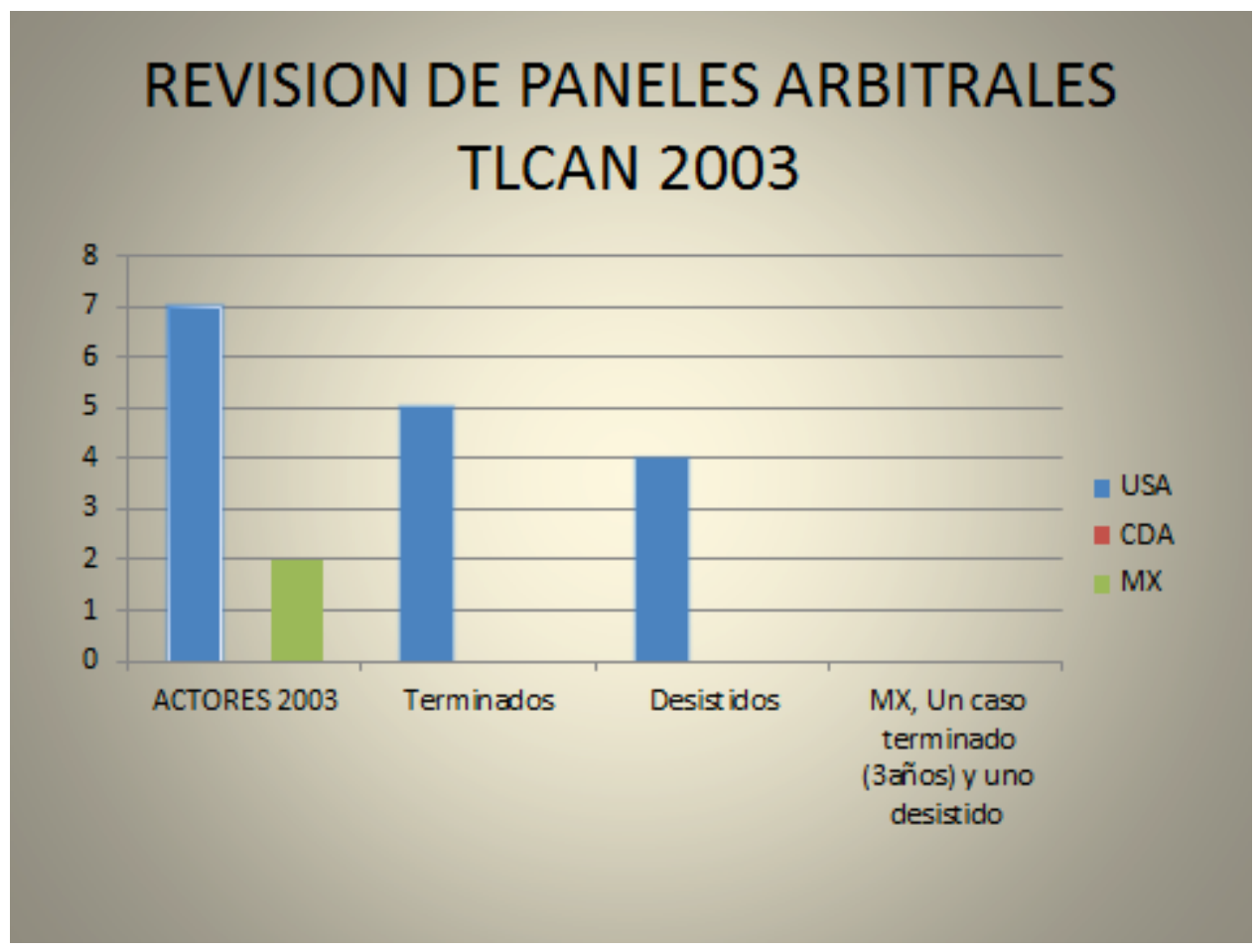




\section{REVISION DE PANELES ARBITRALES}

\section{TLCAN 2004}

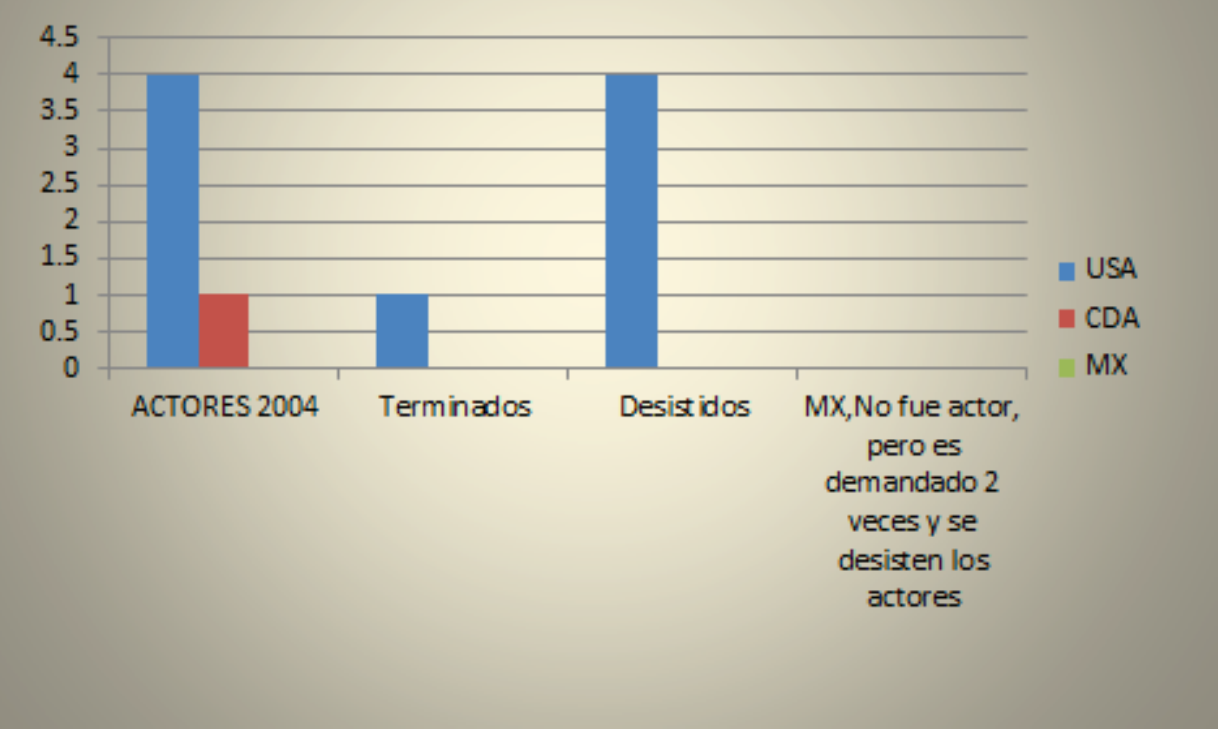

\section{REVISION DE PANELES ARBITRALES} TLCAN 2005

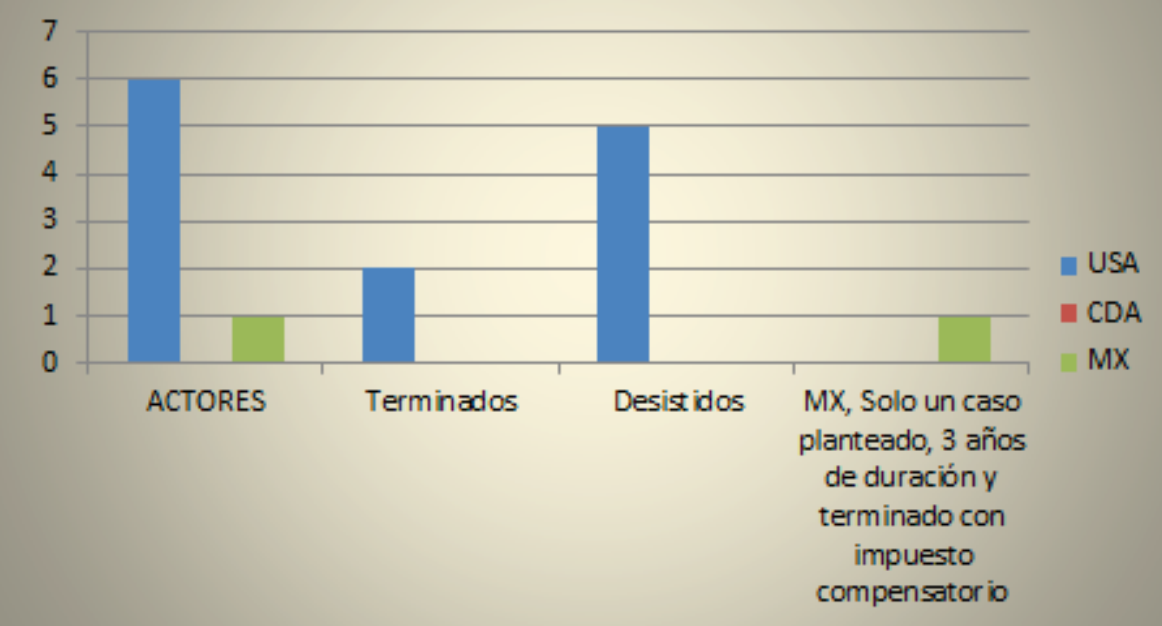




\section{REVISION DE PANELES ARBITRALES TLCAN 2006}

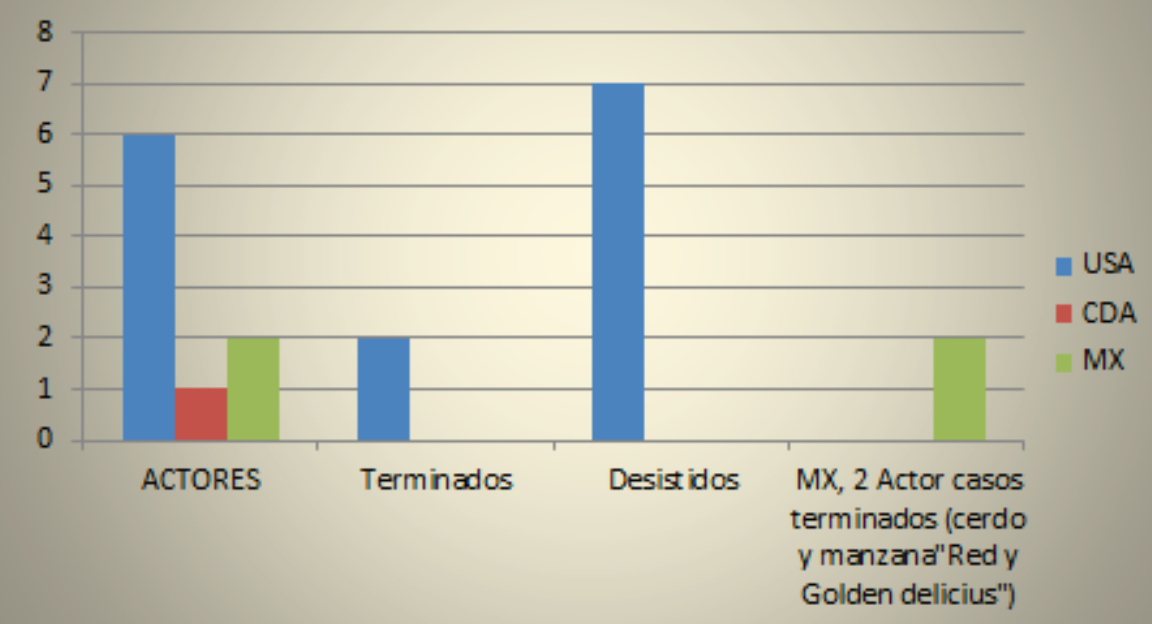

\section{REVISION DE PANELES ARBITRALES TLCAN 2007}

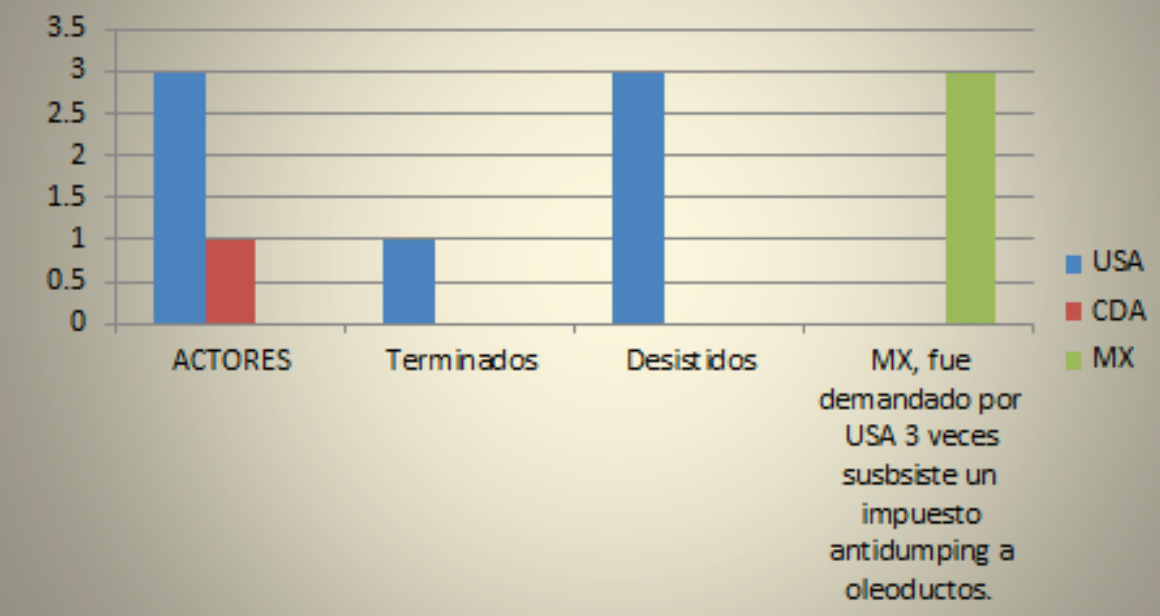




\section{REVISION DE PANELES ARBITRALES TLCAN 2008}

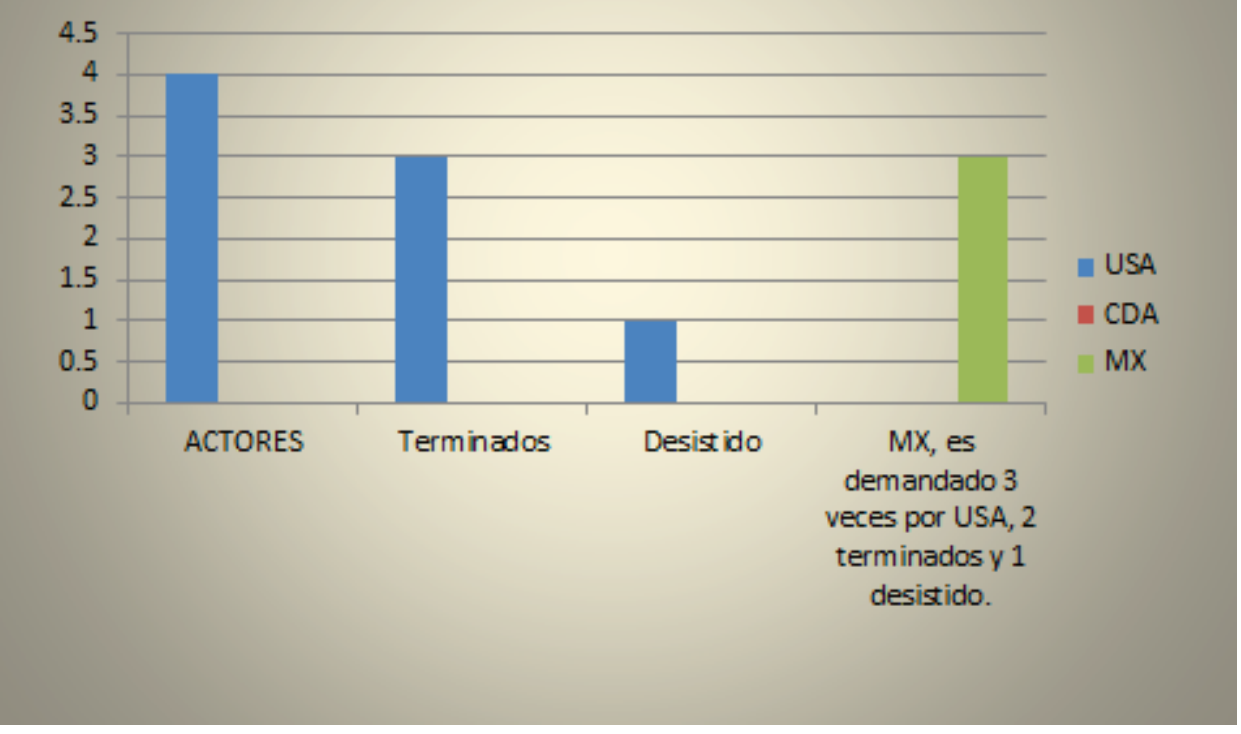

\section{REVISION DE PANELES ARBITRALES} TLCAN 2009

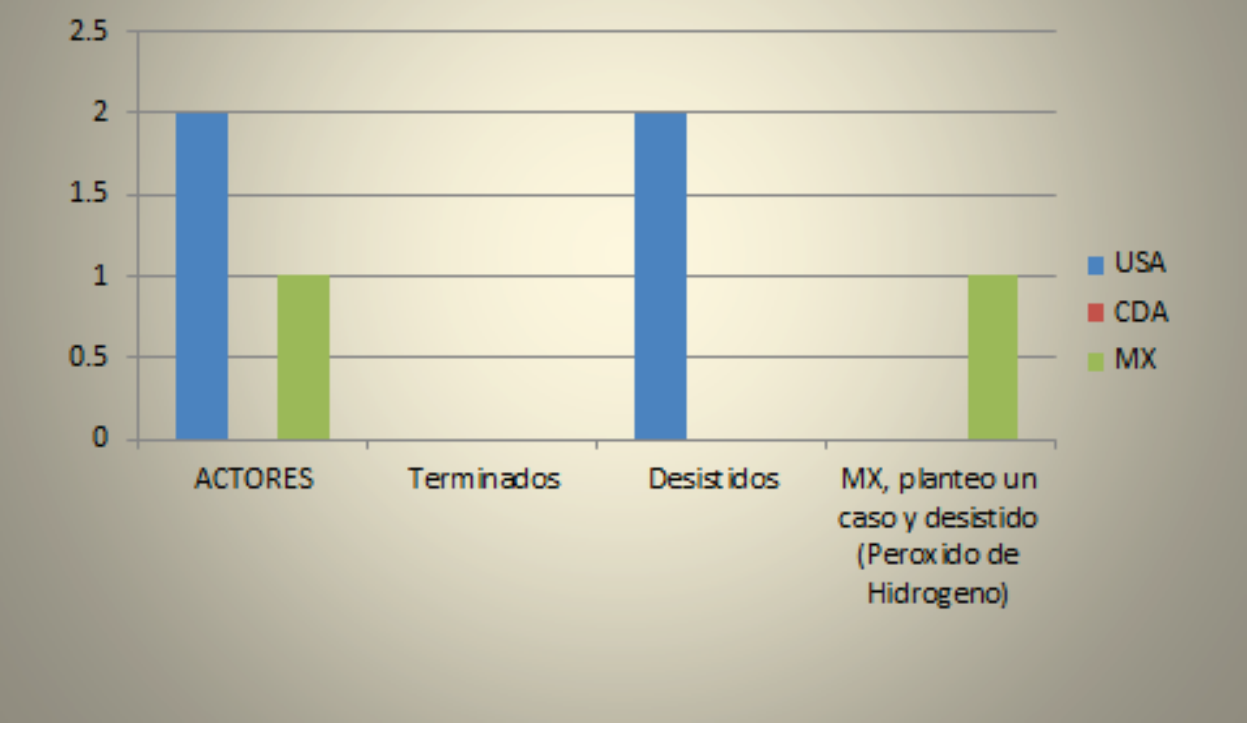




\section{REVISION DE PANELES ARBITRALES} TLCAN 2010
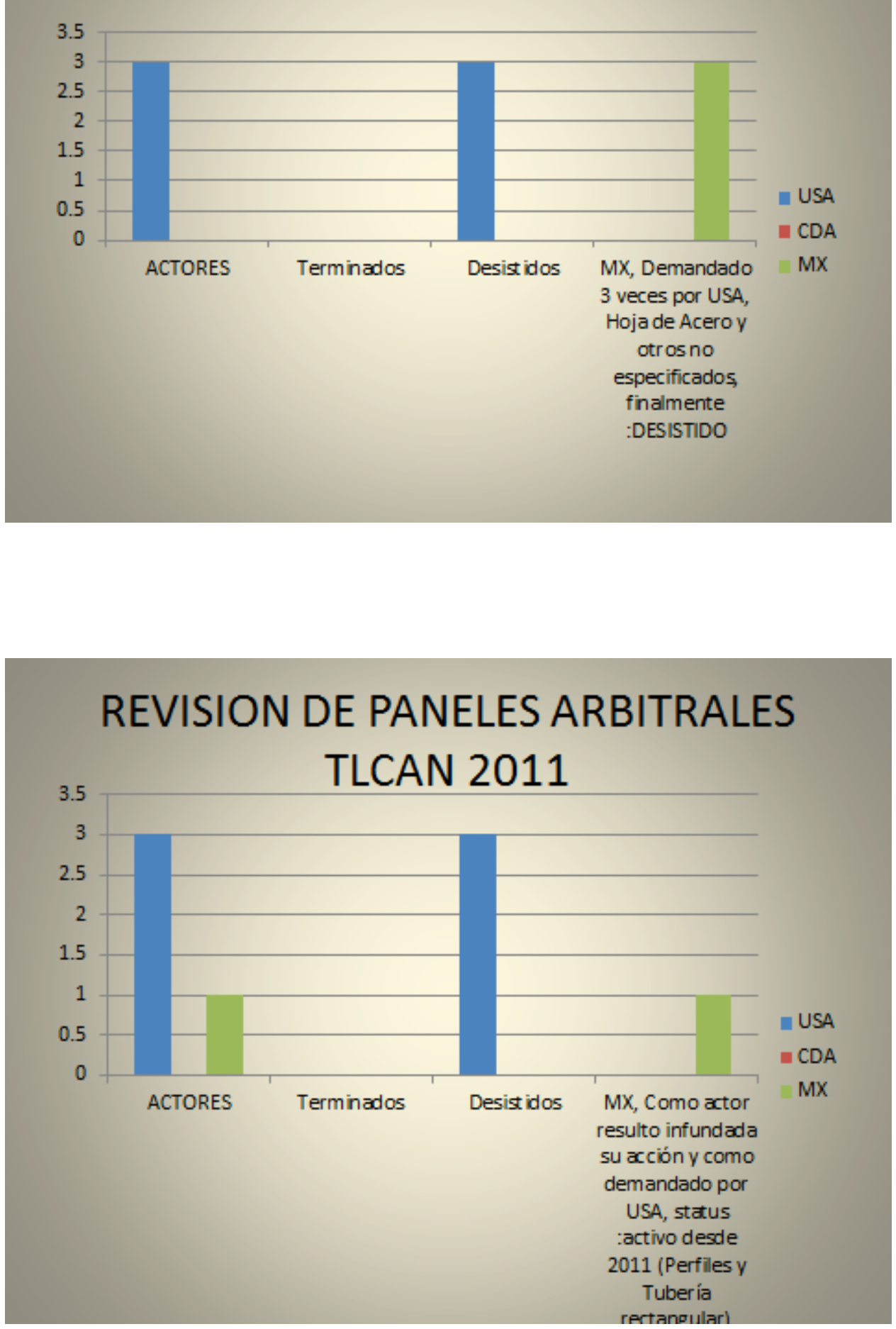


\section{REVISION DE PANELES ARBITRALES TLCAN 2012}

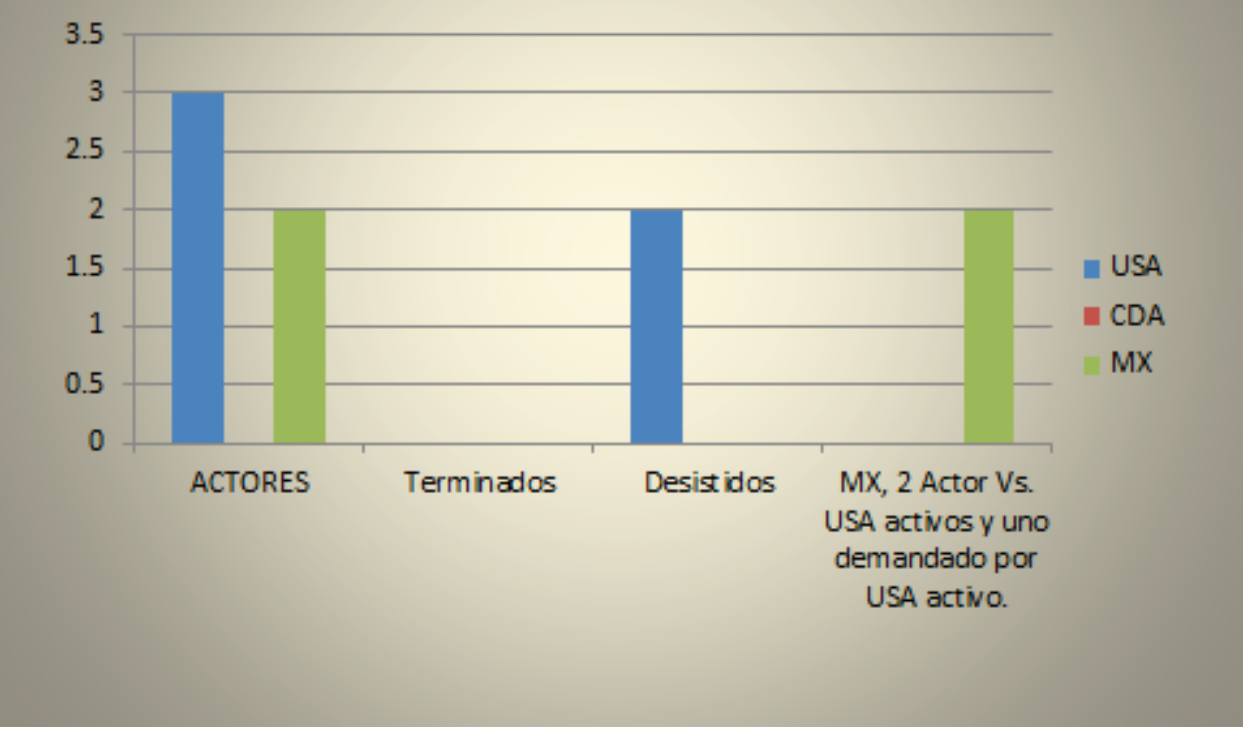




\section{ACTUALIDAD}

\section{REVISION DE LOS PANELES ARBITRALES 2013-2015}

Se encontraron 3 casos cuotas compensatorias o

$$
\text { dumping: }
$$

MX-USA (10-10-2013) ácido esteárico

USA-MX (08-06-2013) tubería acero

USA-CDA (04-29-2014) alambrón de hierro

En los cuales USA, parece que se confirma al no perder caso alguno....!

\section{LISTA BIENES OBJETO DE MASC}

- sosa caustica USA

- manzanas USA

- Pierna y muslo de pollo USA

- Trigo CDA

- Magnesio CDA

- Cemento portland(MX)

- Alambrón de hierro CDA

- Productos de madera suave CDA

- Carboximetilcelulosa (MX

- Productotubulares petrolíferos MX

- Perfiles y tubería rectangular MX
- Persianas venecianas $\mathrm{MX}$

- Chapas y flejes de acero MX

- Luces y tubos MX

- Tubería de cobre

- Oleoducto soldado MX

- Peróxido de hidrógeno

- Refrigeradores/congeladores $\mathrm{MX}$

- Hojas de acero inoxidable MX

- Hoja de acero rollos MX

- Cobrey tubos MX 


\section{LITIGIO EN 10 AÑOS DEL TLC}

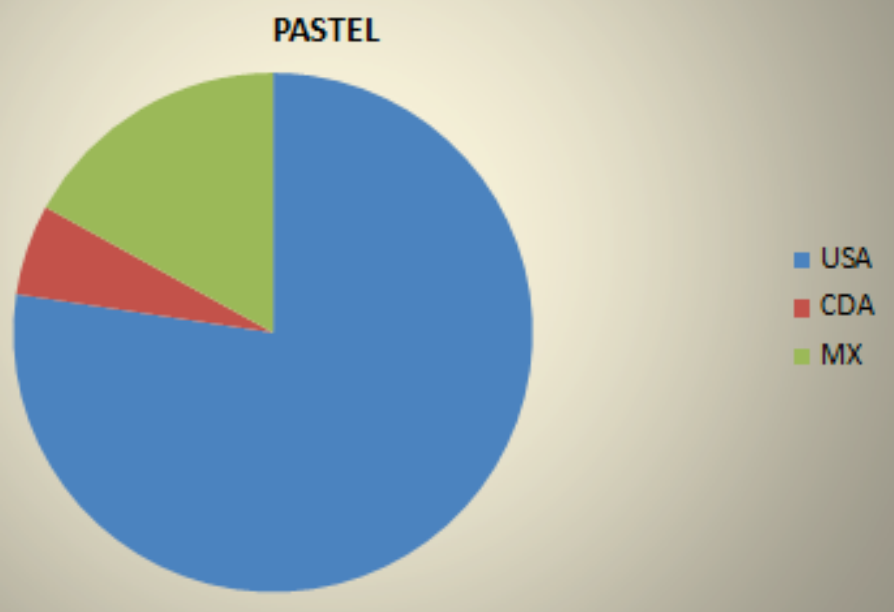

Conclusiones de las Decisiones Arbitrales

A lo largo de 10 años los MASC, han sido accionados por una de las partes preferentemente (USA), lo que significa un instrumento de litigación : ad hoc

Son pocos los sectores en los cuales México es competitivo y ahí USA, presenta reclamaciones con una solo intención: Litigación y tiempo

Es evidente a quien favorece $\mathrm{y} / \mathrm{o}$ quien utiliza los mecanismos alternativos de solución de controversias consecuentemente permea azul (USA), en todas y cada una de las láminas y graficas que les presentamos. 
Hoy día se habla de una nueva negociación de sectores, buen tiempo para ajustar la maquinaria ya que el sector energético ha sido liberado y con ello una posibilidad de obtener beneficios a corto plazo.

Ningún tratado debe ser para siempre y eso se pretendía implementar; estamos ante el juicio de la historia.

\section{Bibliografía y Webgrafía}

- Tratado de Libre Comercio de América del Norte (TLCAN)

Capítulo XI: Inversión, Capítulo XIX: Revisión y solución de controversias en materia de cuotas antidumping y compensatorias, Capítulo XX: Disposiciones institucionales y procedimientos para la solución de controversias. [online]Disponible en: : https://www.nafta-secalena.org/Inicio/Textos-juridicos/Tratado-de-Libre-Comercio-de-América-delNorte?mvid=1\&secid=539c50ef-51c1-489b-808b-9e20c9872d25 [consultado el 15/02/2015] 